\title{
Generation of monochromatic electrostatic waves of large amplitude in a bounded beam-plasma system
}

\author{
By M. BITTER AND P. J. PARIS \\ Centre de Recherches en Physique des Plasmas, \\ Ecole Polytechnique Fédérale de Lausanne, Switzerland
}

(Received 7 January 1975 and in revised form 24 May 1975)

\begin{abstract}
Monochromatic electrostatic waves of large amplitude were excited by the interaction of an electron beam with a bounded plasma. These waves were identified as resonant beam modes, which are amplified by multiple reflexion in a cavity. Nonlinear effects, such as the generation of harmonics and sidebands, were observed.
\end{abstract}

\section{Introduction}

The propagation of single electrostatic waves of large amplitude in a plasma gives rise to a number of nonlinear effects, which have aroused considerable interest in the last few years. The main topics of research in this field are parametric decay of waves and nonlinear effects involved in particle trapping (Franklin et al. 1971 $a, b$; Porkolab 1972, 1974). These include saturation of exponential damping and growth (O'Neil 1965; Wharton, Malmberg \& O'Neil 1968; O'Neil \& Winfrey 1972; Gentle \& Lohr 1973) and excitation of sideband instabilities (Wharton et al. 1968; Franklin et al. 1972; van Hoven \& Jahns 1975; Kruer, Dawson \& Sudan 1969; Mima \& Nishikawa 1971; Brinca 1972).

Experimental studies have been made by exciting electrostatic waves of large amplitude either externally, with antennas (Franklin et al. 1971a, b; Wharton et al. 1968; Franklin et al. 1972) or internally, through the interaction of an electron beam with a plasma (Gentle \& Lohr 1973; van Wakeren \& Hopman 1972). In the latter case, the large-amplitude wave was the most unstable mode that grew out of the background noise after a certain length of interaction with the plasma. This process is described in detail by the single-wave theory of O'Neil \& Winfrey (1972). But the conditions, under which the theory applies, are difficult to obtain experimentally.

We studicd a method of exciting monochromatic electrostatic waves, by the interaction of an electron beam with a plasma within a resonant cavity. The cavity is placed at one end of a cylindrically-bounded steady-state plasma, and also serves as a source for a continuous low-energy electron beam, which is used to produce the plasma. The electron velocity distribution is unstable under certain experimental conditions, and leads to the excitation of electrostatic waves. These are amplified by multiple reflexions in the cavity, if the wavelengths fulfill a condition of resonance. The amplification can be so strong that harmonics of the frequency of an unstable mode are also excited by nonlinear effects. 
A fraction of the energy of the standing waves formed in the cavity is transmitted to the main part of the plasma column, giving rise to a propagating largeamplitude wave. The intensity of these waves is typically $40-60 \mathrm{~dB}$ above the noise level, and the spectral resolution is about $1 \%$. This leads to a trapping of beam electrons, and to the excitation of sideband instabilities in some cases.

Our experimental results confirm some of the observations that Hall $(1972 a, b)$ reported. An important additional result is the observation of harmonics and sidebands. Moreover, a measurement of the dispersion relation and an experimental verification of the threshold criterion help clear up some points in Hall's interpretation of the nature of the excited waves.

\section{Experiment}

\subsection{Apparatus}

The experiments were performed in a steady-state argon plasma, which was produced in a hot cathode glow discharge (figure 1). Electrons emitted from the cathode were accelerated by a potential difference, applied between the cathode and a coarse grid of $80 \%$ transmission $32 \mathrm{~mm}$ in front of the cathode. These produce a plasma, by ionizing collisions with the neutral atoms, with an electron density of about $1 \times 10^{9} \mathrm{~cm}^{-3}$ and electron temperatures of about $5 \mathrm{eV}$. (The plasma was weakly ionized. But the electron-neutral collision frequency $\nu_{e n}$ was only $5 \%$ of the electron plasma frequency $\nu_{p e}$, which was sufficiently small not to effect the growth rate of the unstable modes: Briggs 1971.)

The plasma could be radially confined within a column of the cathode diameter of $5 \mathrm{~cm}$ by a homogeneous magnetic field, which was variable up to $3 \mathrm{k}$ Gauss. In the absence of a magnetic field, it extended over the cross section of the glass tube of $15 \mathrm{~cm}$ diameter. The length of the column was variable up to $85 \mathrm{~cm}$, by a longitudinally movable anode.

Diagnostics were performed using radially and axially movable Langmuir probes, and by measuring the propagation properties of Bernstein waves (Bitter \& Paris 1974). The axial density profile was constant to within $5 \%$ over the central part of the column of $60 \mathrm{~cm}$, and varied by about $20 \%$ over a distance of $10 \mathrm{~cm}$ at the boundaries.

\subsection{Natural modes of the system}

Owing to the variation of the potential along the plasma column, one obtains a double-humped electron velocity distribution with a major peak at a plasma potential of about $5 \mathrm{~V}$, and another peak at a cathode potential representing the fast primary electrons.

The natural modes of the system (in particular, the spectrum of unstable modes) depend on the details of the distribution. Different dispersion relations arise for a 'gentle bump in the tail' distribution, or a distribution theoretically treatable by the cold-beam approximation (O'Neil \& Malmberg 1968).

We measured the dispersion relation, to find out which of these two theoretical models applied to our experiment. The measurements were carried out by means of conventional interferometer techniques (Barrett, Jones \& Franklin 1968; 
(i) (ii) (iii)

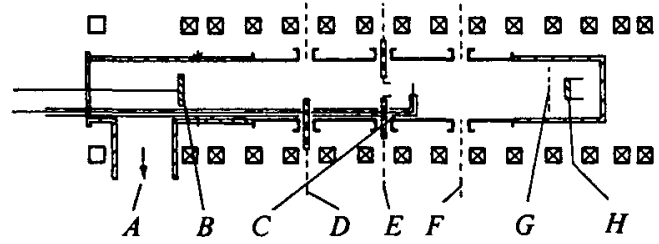

(a)
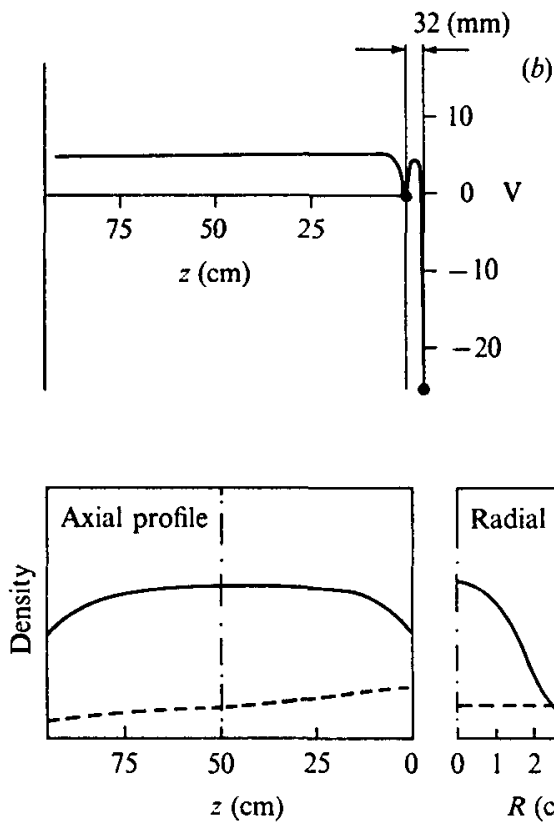

$(c)$
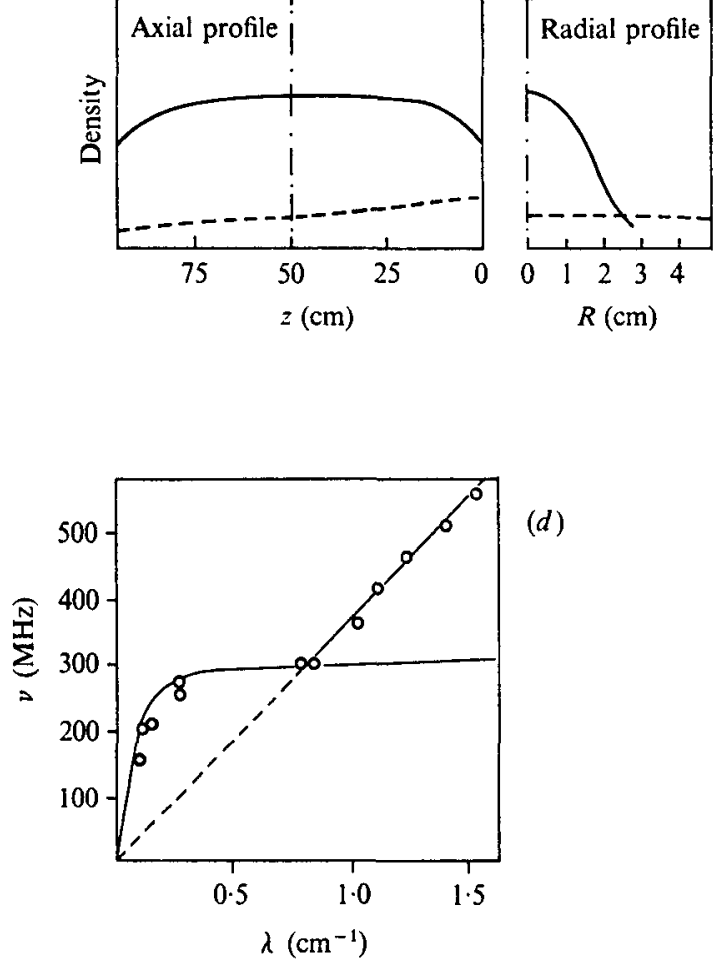

(d)

FraURe 1. (a) Experimental device : (i)-(iii) ports, $A$ pump, $B$ anode, $C$ longitudinal probe, $D$ Langmuir probe, $E$ Bernstein probes, $F$ gas inlet, $G$ grid, $H$ cathode. (b) Axial and radial density profiles with and without magnetic field for $I_{D}=300 \mathrm{~mA}$. (c) Variation of space potential with distance along plasma column. $(d)$ Experimental points of dispersion relation for $I_{D}=280 \mathrm{~mA}, V_{D}=25 \mathrm{~V}, \nu_{c e}=250 \mathrm{MHz}, \nu_{p e} \approx 425 \mathrm{MHz}$. These fall close to the theoretical dispersion curve for the fundamental Trivelpiece-Gould modes at lower frequencies, and follow a straight dispersion line, which agrees well with that expected for the beam modes, at higher frequencies. (Dispersion curve for the Trivelpiece-Gould modes was obtained, approximating the radial density profile by 
Malmberg \& Wharton 1969; Franklin et al. 1974), using Langmuir probes as transmitter and receiver of CW RF signals. Figure 1 shows results obtained for typical experimental conditions. The precision of the wavelength measurement at low frequencies was affected by the density gradient. But one may see clearly in figure 1 that the experimental points are well approximated by a TrivelpieceGould $(1959 a, b)$ mode at lower frequencies, and bend over to a straight dispersion line at higher frequencies. The slope of the straight dispersion line agrees well with the value of the beam velocity calculated from the difference between plasma and cathode potential. This indicates that the cold-beam approximation is a valid description of the system.

Briggs $(1964,1971)$ developed the theory of the interaction of a weak cold electron beam with a plasma. The stability of the space charge modes may be discussed by means of the dispersion relation

$$
\epsilon_{p}(\omega, k)=\frac{\omega_{p b}^{2}}{\left(\omega-k v_{b}\right)^{2}}
$$

$\epsilon_{p}(\omega, k)$ is the dielectric of the plasma; $\omega_{p b}$ is the electron plasma frequency of the beam; $v_{b}$ is the beam velocity. Equation (1) yields two classes of solutions for $\omega \neq k v_{b}$ and for $\omega \approx k v_{b}$, which may be interpreted as the natural modes of the plasma (Trivelpiece-Gould modes in the case of a cylindrically-bounded plasma) and as the slow- and fast-beam modes, respectively. According to (1), the slow-beam modes are unstable for a range of values of $\omega$ and $k$, where $\epsilon_{p}(\omega, k) \leqslant 0$. Because $\omega \approx k v_{b}$, this fact can be expressed as a threshold criterion for the beam velocity $v_{b}$. The threshold criterion assumes a simple form for a plasma-filled waveguide:

$$
v_{b} \leqslant \omega_{p} a / 2 \cdot 4
$$

$\omega_{p}$ and $a$ are the electron plasma frequency, and the radius of the plasma column, respectively.

The growth rate is maximum for $\epsilon_{p} \approx 0$ (i.e. for the synchronized slow-beam and plasma mode). But the growth rate is also appreciable for asynchronous modes.

Fradre 2. Measurement of spectral density and wavelengths of spontaneously excited modes: $(a)$ without a magnetic field $v_{c e}$ and $(b)$ with one. Wave power is measured on a linear scale in arbitrary units. Wavelengths are determined from variation of floating potential of a longitudinally movable Langmuir probe with distance from grid, using a tuned receiver. Measured signal is the result of the interference of a propagating electro. static wave with a direct pick-up signal, which is coupled by a capacitor to the probe

\begin{tabular}{|c|c|c|c|c|c|c|c|c|c|}
\hline & $\begin{array}{r}10^{4} \\
\mathrm{Hg})\end{array}$ & $\begin{array}{c}I_{D} \\
(\mathrm{~mA})\end{array}$ & $\begin{array}{l}V_{D}^{\prime} \\
(\mathrm{V})\end{array}$ & $\begin{array}{c}v_{c e} \\
(\mathrm{MHz})\end{array}$ & (i) & (ii) & (iii) & (iv) & (v) \\
\hline (a) & 3 & 300 & 60 & $\left.{ }^{0}\right\} \begin{array}{l}f(\mathrm{MHz}) \\
\lambda(\mathrm{cm})\end{array}$ & $\begin{array}{l}608 \\
0 \cdot 8\end{array}$ & $\begin{array}{r}532 \\
0.9\end{array}$ & $\begin{array}{l}305 \\
1.6\end{array}$ & $\begin{array}{l}226 \\
2 \cdot 3\end{array}$ & 152 \\
\hline$(b)$ & 4 & 300 & 25 & $\left.{ }^{270}\right\} \begin{array}{l}f(\mathrm{MHz}) \\
\lambda(\mathrm{cm})\end{array}$ & $\begin{array}{r}242 \\
1 \cdot 6\end{array}$ & $\begin{array}{r}484 \\
0.8\end{array}$ & $\begin{array}{l}726 \\
0.6\end{array}$ & - & - \\
\hline
\end{tabular}
from the cathode. 
Waves in bounded plasma
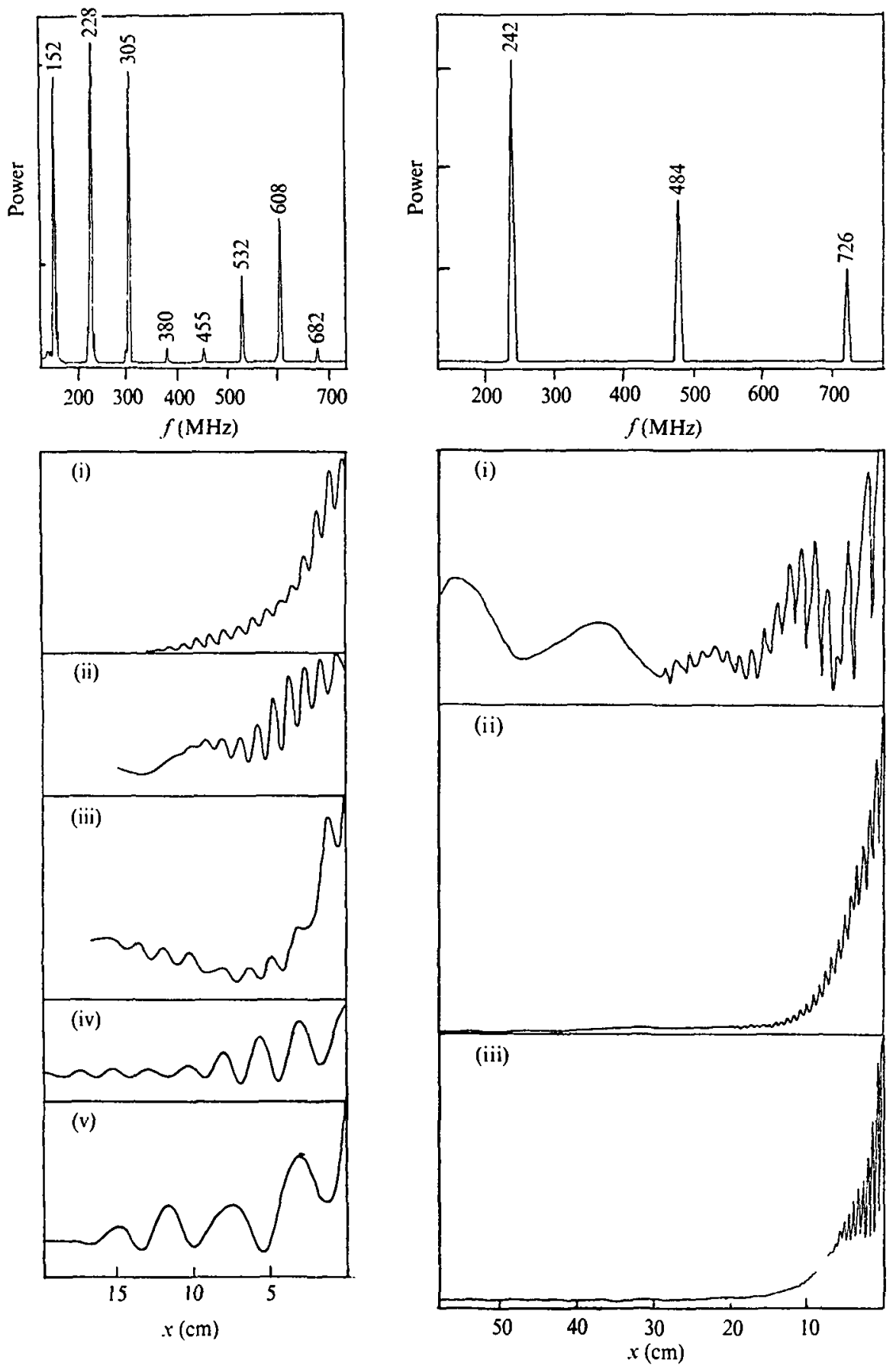

(a)

(b)

Figure 2. For legend see facing page. 
(This would be different for a 'gentle bump in the tail' distribution. Here only certain originally damped modes of the plasma are unstable, owing to the slight deformation of the velocity distribution.)

\subsection{Excitation of large-amplitude waves}

The cathode grid region acts as a resonance cavity for certain unstable beam modes, whose wavelengths are half an integer fraction of the cathode grid distance $(\lambda=64 / n \mathrm{~nm})$. These modes are amplified by mutiple reflexions from the grid and the cathode. But a part of the wave energy is always transmitted through the grid, giving rise to a propagating wave between grid and anode.

Figure 2 shows the spectral density of the modes observed for cases with and without magnetic field. (The magnetic field is expressed in terms of the electron cyclotron frequency $\nu_{c e}$.) The spectrum consists of a set of narrow lines, occurring at the harmonics of a fundamental frequency, which is well removed from the characteristic frequencies $\nu_{c e}, \nu_{p e}$ of the plasma.

Wavelengths were determined by measuring variation of the floating potential of a Langmuir probe with distance from the grid, with a tuned receiver. The measured signal is the result of an interference of the wave electric field, which propagates through the plasma, with a direct pick-up signal, which is coupled by a capacitor to the probe from the cathode. The direct pick-up signal decays over a distance of $15 \mathrm{~cm}$ away from the grid. The remaining long-scale amplitude oscillations, observable for one of the examples of figure 2, are an indication of nonlinear effects resulting from trapping of beam electrons. (In some cases, we observed the excitation of sidebands at neighbouring frequencies. These frequencies could be explained in terms of the electron bounce frequency, which was calculated from the measured values of the wavelength and the wave potential. The wave potential was estimated to be about $500 \mathrm{mV}$, taking into account that only a few percent of the wave power is coupled to the measuring antenna (Franklin et al. 1974). Bitter \& Paris (1975) reported these results.)

The spatial variation of the wavelength, observed for the lower-frequency modes in figure 2 , is obviously an effect of the density gradient. The wavelengths belonging to the higher harmonics, which are well above the electron plasma. frequency, are independent of the plasma density, and agree well with the value indicated above.

The phase velocity derived from the measurements shown in figure 2 is close to the value of the beam velocity. Therefore, the observed waves are beam modes. The measurements described in $\$ 2.4$ constitute further evidence of this.

\subsection{Experimental verification of the threshold criterion}

The parameters entering in (2) cannot be varied independently in our experiment. The relations between the discharge voltage, which is a measure of the beam velocity, and the plasma density can be found from the diagram, figure $3(a)$. A calibration of the measured ion saturation current is obtained from the density measurements with the Bernstein waves in table 1. (The magnetic field and the plasma density are in units of electron cyclotron $\nu_{c e}$, and electron plasma frequency $\nu_{p e}$, respectively). 


\begin{tabular}{|c|c|c|}
\hline$I_{D}(\mathrm{~A})$ & $\begin{array}{c}\nu_{c \varepsilon} \\
\left(\times 10^{8} \mathrm{~Hz}\right)\end{array}$ & $\begin{array}{c}\nu_{v e} \\
\left(\times 10^{8} \mathrm{~Hz}\right)\end{array}$ \\
\hline $0 \cdot 100$ & $3 \cdot 40$ & $3 \cdot 5$ \\
\hline 0.135 & $2 \cdot 95$ & $3 \cdot 8$ \\
\hline $0 \cdot 165$ & $2 \cdot 20$ & $4 \cdot 3$ \\
\hline 0.200 & 1.90 & $4 \cdot 4$ \\
\hline 0.242 & $1 \cdot 30$ & $4 \cdot 6$ \\
\hline 0.270 & 1.00 & $4 \cdot 7$ \\
\hline
\end{tabular}

Table 1.

We infer from figure $3(a)$, that the best procedure, for an experimental verifcation of the threshold criterion, is to measure the intensity of the excited waves as a function of the magnetic field $v_{c e}$ for a fixed discharge current $I_{D}$, since the discharge voltage $V_{D}$ and the plasma density $I_{\text {ion }}^{\text {sat }}$ are changing in an opposite sense under these conditions. The experimental results obtained in this way are shown in figure $3(c)$. The threshold is passed for a certain value of the magnetic field ( $\left.\nu_{c e}=250 \mathrm{MHz}\right)$.

We observe also that the frequency of the excited wave is shifted to lower values, if the magnetic field is increased above the threshold. This behaviour can be explained by figure $3(b)$, which shows qualitatively the fundamental Trivelpiece-Gould mode and the beam mode, for three values of the magnetic field (increasing from 1 to 3 ) and for a given discharge current. This is as expected from figure $3(a)$. That suggests the following interpretation of the experimental results in figure $3(c)$. (i) The frequency of the excited-beam mode $\left(\omega \approx k v_{b}\right)$ is changed through a decrease of beam velocity $v_{b}$, as the $k$ value is fixed by the resonance condition of the cavity. (ii) The excited waves are not synchronous with Trivelpiece-Gould modes, as one would observe an increase of the frequency in this case. (The synchronous modes are represented by the open circles in figure $3(b)$.)

\section{Discussion}

The observations of harmonics and sidebands demonstrate that the apparatus is well suited to the excitation of large-amplitude electrostatic waves, which permit one to investigate various nonlinear effects in a plasma. The determination of the nature of these waves and of the conditions that must be satisfied if they are to be excited, is an important result of this work. It allows us to comment on some points of Hall's interpretation.

(i) Waves excited in the cavity had been tentatively interpreted as TrivelpieceGould modes. We showed, in connexion with an experimental verification of the threshold criterion, that these waves are beam modes and that synchronization with Trivelpiece-Gould modes is not a necessary condition for their excitation.

(ii) The waves observed on the anode side of the grid had been interpreted as standing electromagnetic waves, which were assumed to result from a reflexion at some point within the density gradient. We found that waves excited at the 

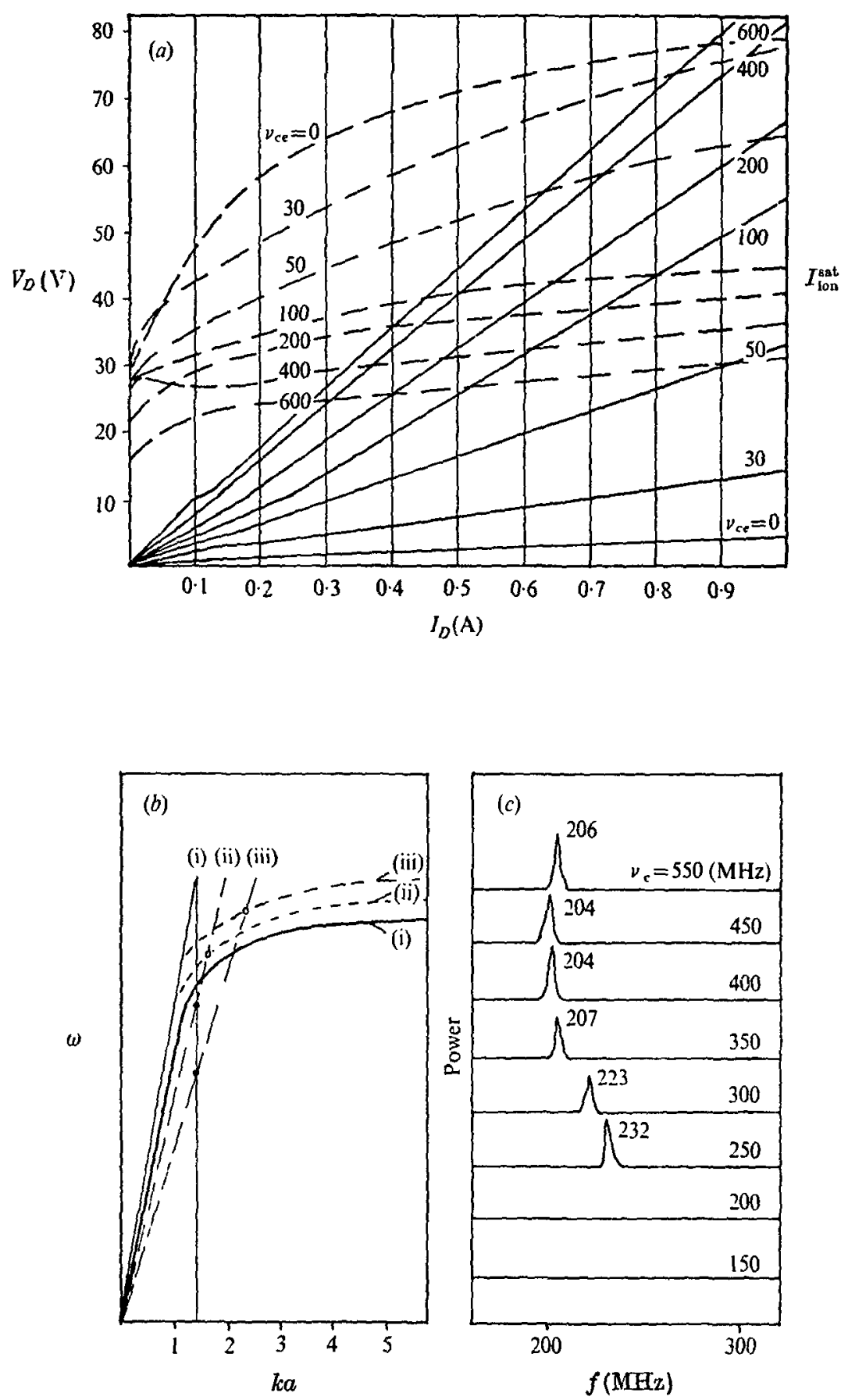

FiGURE 3. (a) Diagram of experimental parameters showing variation of the discharge voltage $V_{D}(\ldots)$ and plasma density, measured by the ion saturation current $I_{j o b}^{\text {sat }}$ taken from a Langmuir probe (-), with the discharge current $I_{D}$ for different values of the magnetic field and a neutral gas pressure of $5 \times 10^{-4} \mathrm{~mm} \mathrm{Hg}$. (b) Measurement of spectral density of excited mode as a function of magnetic field for constant $I_{D}=200 \mathrm{~mA}$. (Wave power measured on a linear scale in arbitrary units.) $\omega_{b}=k v$. Threshold, $v_{b} \leqslant\left(\omega_{p} a\right) / p_{\infty}$. (c) Sketch of dispersion relation for fundamental Trivelpiece-Gould modes and electrostatic beam modes for three values (increasing from (i) to (iii)) of the magnetic field and given discharge current. (As expected for our experiment.) 
harmonics of a fundamental frequency are governed by a linear dispersion curve, the slope of which agrees well with the beam velocity, if one takes directly measured values of the frequencies and wavelengths. (See figure 2.) We conclude that the waves on the anode side of the grid are propagating electrostatic beam modes. A further confirmation is the observation of sidebands, which appeared only on the anode side of the grid, and are caused by the trapping of beam electrons.

The slope of the dispersion line for the waves belonging to the harmonics was found to be equal to the beam velocity in the case without a magnetic field; but it was slightly higher $(\approx 20 \%)$ in the other cases. We think that the discrepancy might be explained, were one to account for a finite temperature of the beam which changes the phase velocity of the waves supported by the beam by about the thermal velocity spread. (The difference of slope cannot be caused by e.g. finite boundaries, or a drift of the plasma against the beam. The effect of the boundaries on the beam modes (at high frequencies) is only a parallel shift of the dispersion line, not a change of slope. A drift of the main plasma is unimportant, because, as the modes are carried by the beam, only the velocity of the beam relative to the laboratory matters. We also point out that the floating potential of the anode was more negative than the cathode potential for almost all experimental conditions. This indicates that the electron beam is not thermalized in the plasma, but reflected from the anode. Thus, there is no reason for a drift of the plasma electrons towards the grid, as required by Hall (1972a), to compensate for a current induced by beam electrons in the anode grid region.) The velocity spread of the beam is larger with than it is without a magnetic field, owing to the pseudo-temperature introduced by the radial variation of the plasma potential (Malmberg \& Wharton 1969). Measurements of the beam-velocity distribution showed a temperature spread of about $1 \mathrm{eV}$. This gives, for typical discharge voltages of 20-30 V, values of about $20 \%$ for $\Delta v / v_{0}$.

We investigated a method of exciting electrostatic waves, by the interaction of an electron beam with a plasma in a resonant cavity. This was efficient with respect to the intensity and monochromacy of the waves, and allowed one to select a single wave by tuning the cavity to the desired wavelength.

We thank Mr R. Gruber and Mr Gno for the computation of the dispersion diagram. Helpful discussions with Dr Nguyen The Hung, Dr F. Troyon, Mr M. Q. Tran and Professor E. S. Weibel are also gratefully acknowledged.

\section{REFERENCES}

Barretr, P. J., Jones, H. G. \& Franklin, R. N. 1968 Plasma Phys. 10, 911.

Bitter, M. \& Paris, P. J. 1974 J. Appl. Math. Phys. 25, 677.

Bitter, M. \& Paris, P. J. 1975 Proc. 2nd Int. Cong. on Waves and Instabilities in Plasmas, Innsbruck (ed. Institut für Theoretische Physic der Universitat Innsbruch), p. 12.

BRIGGS, R. J. 1964 Electron-beam interaction with plasmas. Research Monograph 29. MIT Press.

Briggs, R. J. 1971 Advances in Plasma Physics (ed. A. Simon and W. B. Thompson), vol. 4, p. 43. Wiley.

Brinca, A. L. 1972 J. Plasma Phys. 7, 385. 
Frankutn, R. N., Hamberger, S. M., Ikezi, H., Lampis, G. \& Smith, G. J. 1972 Phys. Rev. Lett. 28, 1114.

Frankuin, R. N., Hamberger, S. M., Lampis, G. \& Smith, G. J. 1971 a Phys. Rev. Lett. 27, 1119.

Frankin, R. N., Hamberaer, S. M., Lampis, G. \& Smith, G. J. 1971 b Phys. Lett. 36 A, 473.

Frankin, R. N., Hamberger, S. M., Lampis, G. \& Smith, G. J. 1974 Culham Laboratory Rep. 131.

Gentle, K. W. \& LoHr, J. 1973 Phys. Fluids 16, 1464.

Hall, T. A. $1972 a$ Proc. 5th European Conf. on Controlled Fusion and Plasma Physics (ed. Service d'Ionique Général, Association Euraton-CEA, centre d'Etudes Nucléaire de Grenoble), p. 158.

HaLl, T. A. $1972 b$ Plasma Phys. 14, 667.

Hoven, G. van \& Jahns, G. 1975 Phys. Fluids 18, 80.

Kruer, W. L., Dawson, J. M. \& Sudan, R. N. 1969 Phys. Rev. Lett. 23, 838.

Malmberg, J. H. \& Wharton, C. B. 1969 Phys. Fluids 13, 2600.

Mrma, K. \& Nishrkawa, K. 1971 J. Phys. Soc. Japan 30, 1722.

O'Neir, T. M. 1965 Phys. Fluids, 8, 2255.

O'Neil, T. M. \& Malmberg, J. H. 1968 Phys. Fluids 11, 1754.

O'NeIL, T. M. \& WrNfreY, J. H. 1972 Phys. Fluids 15, 1514.

Porkolab. M., Arunasalam, V. \& Ellis, R. A. 1972 Phys. Rev. Lett. 29, 1438.

Porkolab, M. 1974 MATT-1049.

Trivelpiece, A. W. \& Gould, R. J. $1959 a$ J. Appl. Phys. 30, 11.

Trivelpiece, A. W. \& Gould, R. J. $1959 b$ J. Appl. Phys. 30, 1784.

Wakeren, J. H. A. van \& Hopman, H. H. 1972 Phys. Rev. Lett. 28, 295.

Wharton, G. B., Malmberg, J. H. \& O'Nerr, T. M. 1968 Phys. Fluids 11, 1761. 